\title{
Prager, Katharina: Berthold Viertel. Eine Biografie der Wiener Moderne, 364 S., Böhlau, Wien u.a. 2018.
}

\section{Thomas Stockinger}

Online publiziert: 4. Mai 2020

(C) Der/die Autor(en) 2020

Der in Wien geborene Theater- und Filmregisseur, Schriftsteller und Dichter Berthold Viertel (1885-1953) ist keine kanonische Gestalt. Bekannt ist er allenfalls in der Forschung zur österreichischen Exilliteratur. Die allgemeine Literaturgeschichte ignoriert ihn ebenso wie die umfangreiche Literatur zur, Wiener Moderne ' oder ,Wien um 1900`. Viertel bietet einen schwierigen, aber lohnenden Gegenstand biografischen Schreibens. Ein Grund dafür sind die vielen Einschnitte seines Lebenswegs, der ihn nach Jahrzehnten zwischen Deutschland, den USA und Großbritannien erst 1948 nach Wien zurückführte. Ein weiterer ist die Komplexität und Bruchstückhaftigkeit des ,,autobiografischen Projekts“, zu dem er ab 1906 immer wieder Anläufe unternahm. Er schrieb eine Vielzahl von Texten und Fragmenten, ohne zu einem Abschluss oder zu einer endgültigen Form zu gelangen. Ziel war nicht nur eine eigene Erfolgs- oder (wie er es sah) Misserfolgsgeschichte, sondern Zeitgeschichte durch die Linse des eigenen Lebens. Vor allem wollte er die Wurzeln der Katastrophen des 20. Jahrhunderts, insbesondere der Weltkriege und des Nationalsozialismus, in den Ambivalenzen der Moderne um 1900 suchen, die er teilnehmend und gestaltend miterlebt hatte. Viertels selbstreflexive Rückschau blieb stets sensibel für die Spannung zwischen kritischer Positionierung und Verstrickung. Sein Bewusstsein dafür, dass die Probleme und „Illusionen“, die er als junger Mann bekämpft hatte und als alter erinnerte, auch Teil von ihm blieben und er Teil von ihnen blieb, hebt die Autorin hervor (S. 25). Sie kontrastiert es mit der (viel breiter rezipierten) nostalgischen Perspektive Stefan Zweigs in „Die Welt von gestern“ (S. 68 f.).

Die eingehende Rezeption der neueren Biografieforschung und der reflektierte Umgang mit den Potenzialen und Risiken biografischen Schreibens zählen zu den großen Stärken dieses Buches. Eine klassische Lebensgeschichte strebt Prager nicht an. Ihr Buch beginnt mit einem „Chronologischen Überblick“ (S. 7-16), den sie als Hilfsmittel, nicht als eigenes Forschungsresultat präsentiert. Die schwierige faktografische Rekonstruktion von Viertels transkontinentalem Leben schreibt sie der Handvoll Forschender zu, die sich ihm bisher gewidmet haben; in etlichen Punkten ist sie aber durch Archivarbeit darüber hinausgelangt. Auf eine dichte theoretische und methodische Einleitung (S. 19-44) folgt eine knappe Darstellung seines Lebenswegs von 1918, als er Österreich verließ, bis 1953 (S. 45-96); einen Schwerpunkt

T. Stockinger $(\square)$

Leibniz-Archiv, Niedersächsische Landesbibliothek, Hannover, Deutschland

E-Mail: thomas.stockinger@gwlb.de 
bildet hier das Aufspüren der Phasen, in denen er aus wechselnden Antrieben sein autobiografisches Projekt aufgriff.

Der Hauptteil des Buches ist eine topisch gegliederte Auswertung des autobiografischen Materials. Die Autorin hat dieses vollständiger erfasst, als je zuvor geschehen konnte, gestützt auf die Nachlässe Viertels und seiner Gattinnen Salka Viertel und Elisabeth Neumann im Deutschen Literaturarchiv Marbach sowie auf viele kleinere Bestände in Österreich, Deutschland und den USA (S. 34-38, 336-341). 15 Abschnitte von je zwischen zehn und 25 Seiten sind einzelnen „Erinnerungsorten der Wiener Moderne“ gewidmet (S. 97-331), die im kulturellen Gedächtnis und in der Forschung zu Wien um 1900 ebenso eine wichtige Rolle spielen wie bei Viertel. Manche Themen sind erwartbar, etwa „Moderne in Wien“, „Luegers Wien“, „Erster Weltkrieg“; andere kommen überraschender, einige Titel gehen auf idiosynkratische Formulierungen Viertels zurück (,Mitschüler Hitler“). Mehrere große Fragen kehren immer wieder: so die Spannung zwischen den um 1900 bereits etablierten Aspekten der Wiener Moderne und jenen jüngeren, kritischen Strömungen, denen Viertel sich zuordnete, den Zwiespalt vorzugsweise als Konstellation aus ,erhaltenden Vätern“ und ,zerstörenden Söhnen“ metaphorisierend (S. 99-117). Zentral ist außerdem sein schwieriger Umgang mit seiner jüdischen Identität - die Familie verkörperte den raschen Übergang von der ,traditionellen ' Lebenswelt der Großeltern in Galizien in das gewerbliche urbane Bürgertum und weiter in die intellektuellen Eliten Wiens. Die von Kindheit an gemachten Erfahrungen mit Antisemitismus figurieren in der Suche nach den Anfangsgründen von Diktatur und Krieg. Pragers Nachforschungen nach Verwandten Viertels enden mehrfach bei deren Ermordung in der Shoah.

In behutsam explizierter Präsentation hält Prager mehrere Wahrnehmungsebenen gegeneinander: Viertels Sicht aus zeitnahen und rückblickenden Aufzeichnungen, von ihm kritisierte dominierende Selbstwahrnehmungen und Gedächtnisformationen sowie Kenntnisstand und Interessen aktueller Forschung. ,Leerstellen“ (S. 41) von Viertels Gesellschaftskritik treten etwa in der Genderperspektive hervor. „Sexuelle Emancipation [sic]“ mochte zu seinen Anliegen zählen (S. 245-267), und er heiratete dreimal gebildete, beruflich erfolgreiche Frauen. Wenn aber die angestrebte offene Ehe auch von der Gattin so gelebt wurde, kam er damit ebenso schlecht zurecht wie mit zeitweiliger ökonomischer Abhängigkeit von deren Einkommen. Verstörend sind jene (kaum zur Publikation gedachten) Notate, in denen er die Vergewaltigung einer Dienstbotin und die Beteiligung an Kinderprostitution im Ersten Weltkrieg festhielt (S. 158f., 324). Der kontextualisierende, nie beschönigende Umgang damit ist kennzeichnend für die Sorgfalt, mit der die Autorin jede Heroisierung oder Dämonisierung ihres Protagonisten vermeidet - obwohl er für beides Material liefern würde.

Selten habe ich ein Buch gelesen, das die eigene Arbeitsweise so konsequent und explizit offenlegte und doch so flüssig und fesselnd zu lesen blieb. Katharina Pragers Arbeit ist eine eindrucksvolle Demonstration des Potenzials einer neuen Biografik, ein wichtiger Beitrag zur Erforschung kollektiver und individueller Gedächtnisarbeit und zugleich ein wertvoller Fortschritt für die Debatten über die Wiener Moderne.

Funding Open Access funding provided by Projekt DEAL. 
Open Access Dieser Artikel wird unter der Creative Commons Namensnennung 4.0 International Lizenz veröffentlicht, welche die Nutzung, Vervielfältigung, Bearbeitung, Verbreitung und Wiedergabe in jeglichem Medium und Format erlaubt, sofern Sie den/die ursprünglichen Autor(en) und die Quelle ordnungsgemäß nennen, einen Link zur Creative Commons Lizenz beifügen und angeben, ob Änderungen vorgenommen wurden.

Die in diesem Artikel enthaltenen Bilder und sonstiges Drittmaterial unterliegen ebenfalls der genannten Creative Commons Lizenz, sofern sich aus der Abbildungslegende nichts anderes ergibt. Sofern das betreffende Material nicht unter der genannten Creative Commons Lizenz steht und die betreffende Handlung nicht nach gesetzlichen Vorschriften erlaubt ist, ist für die oben aufgeführten Weiterverwendungen des Materials die Einwilligung des jeweiligen Rechteinhabers einzuholen.

Weitere Details zur Lizenz entnehmen Sie bitte der Lizenzinformation auf http://creativecommons.org/ licenses/by/4.0/deed.de.

\title{
Gräb, Sebastian: Der Sprachgebrauch paramilitärischer Kampfbünde. Eine Untersuchung zur politisch-ideologischen Kommunikation in der Weimarer Republik, 444 S., Kovač, Hamburg 2018.
}

\author{
Sebastian Elsbach \\ Online publiziert: 30. April 2020 \\ (C) Der/die Autor(en) 2020
}

Die Wehrverbände beziehungsweise „Kampfbünde“ der 1920er und 1930er Jahre stellen in der wissenschaftlichen Rückschau und dem öffentlichen Gedächtnis ein epochemachendes Phänomen dar. Die Straßenkämpfe gelten als zentraler Teil des tödlichen Problemcocktails der Weimarer Republik. Bislang gab es jedoch keine wissenschaftliche Arbeit, die alle wichtigen Verbände vergleichend untersucht. Die vorliegende sprachwissenschaftliche Dissertation von Sebastian Gräb betritt in interdisziplinärer Hinsicht Neuland und möchte sowohl die quantitative Sprachwissenschaft als auch die Forschung zur Weimarer Republik bereichern, was - mit einigen Einschränkungen, auf die einzugehen sein wird - durchaus gelungen ist. Besonders in den Unterkapiteln zur Namenspolemik (Kap. 5.1, 5.2, 5.6) als sprachlicher Technik zur Diffamierung des Gegners und dem Abschnitt zum ,publizistischen Symbolkampf“ (Kap. 5.8) wird der Mehrwert der softwaregestützten Untersuchung

S. Elsbach $(\bowtie)$

Friedrich-Schiller-Universität Jena, Jena, Deutschland

E-Mail: sebastian.elsbach@uni-jena.de 